C. Virdol

Nagoya Math. J.

Vol. 190 (2008), 87-104

\title{
ON $L$-FUNCTIONS OF TWISTED 3-DIMENSIONAL QUATERNIONIC SHIMURA VARIETIES
}

\author{
CRISTIAN VIRDOL
}

\begin{abstract}
In this paper we compute and continue meromorphically to the entire complex plane the zeta functions of twisted quaternionic Shimura varieties of dimension 3. The twist of the quaternionic Shimura varieties is done by a mod $\wp$ representation of the absolute Galois group.
\end{abstract}

\section{$\S 1$. Introduction}

Let $X$ be a smooth projective variety defined over a number field $F$ and let

$$
\bar{X}=X \times_{F} \overline{\mathbb{Q}} \text {. }
$$

For a prime number $l$, let $H_{e t}^{i}\left(X, \overline{\mathbb{Q}}_{l}\right)$ be the l-adic cohomology of $\bar{X}$. If $K$ is a number field, we denote $\Gamma_{K}:=\operatorname{Gal}(\overline{\mathbb{Q}} / K)$. The Galois group $\Gamma_{F}$ acts on $H_{e t}^{i}\left(X, \overline{\mathbb{Q}}_{l}\right)$ by a representation $\rho_{i, l}$. The $L$-function $L^{i}\left(s, X_{/ F}\right)$ attached to the representation $\rho_{i, l}$ converges for $\operatorname{Re}(s)>1+i / 2$. It is conjectured that the $L$-function $L^{i}\left(s, X_{/ F}\right)$ converges and does not vanish in the half plane $\operatorname{Re}(s) \geqslant 1+i / 2$ and it has a meromorphic continuation to the complex plane with a finite number of poles and satisfies a functional equation.

In this article we look at the zeta functions of "twisted" quaternionic Shimura varieties (see below) and in particular general quaternionic Shimura varieties of dimension 3 . We show that their zeta functions can be meromorphically continued to the complex plane, satisfy functional equations, converge and do not vanish in the half plane $\operatorname{Re}(s) \geqslant 5 / 2$. To obtain these results, we use in particular the computation of all the local factors of the $L$-functions of quaternionic Shimura varieties (see [B, Theorem 3]) and the Weight-Monodromy conjecture that is known in the case of quaternionic Shimura varieties (see [B, Theorem 2]). We prove all these results also for the base change of these Shimura varieties to arbitrary solvable extensions of totally real fields that contain their field of definition (see below).

Received July 14, 2006.

2000 Mathematics Subject Classification: 11F03, 11F41, 11F80, 11R37, 11R42, 11R52, 11R56, 11R $80,11 \mathrm{~S} 40$. 
More exactly, in this paper we consider a totally real field $F$ with $[F$ : $\mathbb{Q}] \geqslant 3$ and a quaternion algebra $D$ over $F$, which is unramified at exactly 3 of the infinite places of $F$. Let $G$ be the algebraic group over $F$ defined by the multiplicative group $D^{\times}$of $D$ and let $\bar{G}=\operatorname{Res}_{F / \mathbb{Q}}(G)$. For $\wp$ a prime ideal of the ring of integers $O:=O_{F}$ of $F$, such that $G\left(F_{\wp}\right)$ is isomorphic to $G L_{2}\left(F_{\wp}\right)$, let $S_{\bar{G}, \mathbf{K}}=S_{\mathbf{K}}$ be the canonical model of the quaternionic Shimura variety associated to an open compact subgroup $\mathbf{K}:=K_{\wp} \times H$ of $\bar{G}\left(\mathbb{A}_{f}\right)$, where $K_{\wp}$ is the set of elements of $G L_{2}\left(O_{\wp}\right)$ that are congruent to 1 modulo $\wp, H$ is an open compact subgroup of the restricted product of $\left(D \otimes_{F} F_{\mathfrak{p}}\right)^{\times}$where $\mathfrak{p}$ runs over all the finite places of $F, \mathfrak{p} \neq \wp$ and $\mathbb{A}_{f}$ is the finite part of the ring of adeles $\mathbb{A}_{\mathbb{Q}}$ of $\mathbb{Q}$. Then $S_{\mathbf{K}}$ is a quasi-projective variety defined over a totally real finite extension $E / \mathbb{Q}$ called the canonical field of definition.

The variety $S_{\mathbf{K}}$ has a natural action of $G L_{2}(O / \wp)$. For $H$ sufficiently small this action is free. We fix such a small group $H$ and consider a continuous Galois representation $\varphi: \Gamma_{E} \rightarrow G L_{2}(O / \wp)$ and let $S_{\mathbf{K}}^{\prime}$ be the variety defined over $E$ obtained from $S_{\mathbf{K}}$ via twisting by $\varphi$ composed with the natural action of $G L_{2}(O / \wp)$ on $S_{\mathbf{K}}$ (see Section 2 for details).

We assume that $D \neq M_{2}(F)$ and $L:=\overline{\mathbb{Q}}^{\operatorname{Ker}(\varphi)}$ is a solvable extension of a totally real field, and in this case we prove that (see Theorem 6.1) if $k$ is an arbitrary solvable extension of a totally real field containing $E$, then the $L$-function $L^{3}\left(s, S_{\mathbf{K} / k}^{\prime}\right)$ is holomorphic and does not vanish in the half plane $\operatorname{Re}(s) \geqslant 5 / 2$ and satisfies a functional equation. We remark that one can obtain these results also for general (not twisted) quaternionic Shimura varieties of arbitrary level and also for more general twisted quaternionic Shimura varieties (see Remark 6.2).

\section{§2. Twisted quaternionic Shimura varieties}

Let $F$ be a totally real field of degree $d \geqslant 3$ over $\mathbb{Q}$ and $O:=O_{F}$ be its the ring of integers. Let $\mathbb{A}_{\mathbb{Q}}=\mathbb{R} \times \mathbb{A}_{f}$ be the ring of adeles of $\mathbb{Q}$ and $\mathbb{A}_{F}$ the ring of adeles of $F$. We denote by $I_{\mathbb{Q}}$ and $I_{F}$ the groups of ideles of $\mathbb{Q}$ and $F$, respectively.

We consider a quaternion algebra $D$ over $F$ which is unramified at exactly 3 infinite places of $F$. We denote by $S_{\infty}$ the set of the infinite places

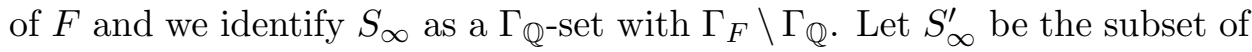
$S_{\infty}$ at which $D$ is ramified. Thus the cardinality of $S_{\infty}-S_{\infty}^{\prime}$ is equal to 3 .

Let $G$ be the algebraic group over $F$ defined by the multiplicative group $D^{\times}$. By restricting the scalars, we obtain the algebraic group $\bar{G}=$ 
$\operatorname{Res}_{F / \mathbb{Q}}(G)$ over $\mathbb{Q}$ defined by the propriety: $\bar{G}(A)=G\left(A \otimes_{\mathbb{Q}} F\right)$ for all $\mathbb{Q}$ algebras $A$. It is easy to see that $\bar{G}(\mathbb{R})$ is isomorphic to $G L_{2}(\mathbb{R})^{3} \times \mathbf{H}^{\times(d-3)}$, where $\mathbf{H}$ is the algebra of quaternions over $\mathbb{R}$.

For $v \in S_{\infty}-S_{\infty}^{\prime}$, we fix an isomorphism of $G\left(F_{v}\right)$ with $G L_{2}(\mathbb{R})$. We have $\bar{G}(\mathbb{R})=\prod_{v \in S_{\infty}} G\left(F_{v}\right)$. Let $J=\left(J_{v}\right) \in \bar{G}(\mathbb{R})$, where

$$
J_{v}= \begin{cases}1 & \text { for } v \in S_{\infty}^{\prime} \\
1 / \sqrt{2}\left(\begin{array}{cc}
1 & 1 \\
-1 & 1
\end{array}\right) & \text { for } v \in S_{\infty}-S_{\infty}^{\prime} .\end{cases}
$$

Let $K_{\infty}$ be the centralizer of $J$ in $\bar{G}(\mathbb{R})$. Set

$$
X=\bar{G}(\mathbb{R}) / K_{\infty}
$$

It is well known that $X$ is complex analytically isomorphic to $\left(\mathcal{H}_{ \pm}\right)^{3}$ where $\mathcal{H}_{ \pm}=\mathbb{C}-\mathbb{R}$. For each open compact subgroup $K \subseteq \bar{G}\left(\mathbb{A}_{f}\right)$ set

$$
S_{K}(\mathbb{C})=\bar{G}(\mathbb{Q}) \backslash X \times \bar{G}\left(\mathbb{A}_{f}\right) / K \text {. }
$$

For $K$ sufficiently small, $S_{K}(\mathbb{C})$ is a complex manifold which is the set of the complex points of a quasi-projective variety. In general $S_{K}(\mathbb{C})$ is not connected. The canonical field of definition of $S_{K}(\mathbb{C})$ is by definition the subfield $E$ of $\overline{\mathbb{Q}}$ such that $\Gamma_{E}$ is the stabilizer of $S_{\infty}^{\prime} \subseteq \Gamma_{F} \backslash \Gamma_{\mathbb{Q}}$. It is known that $S_{K}(\mathbb{C})$ has a canonical model over the totally real field $E$ which is denoted by $S_{K}$. The dimension of $S_{K}$ is equal to 3 .

Let $\wp$ be a prime ideal of $O_{F}$ such that $G\left(F_{\wp}\right)$ is isomorphic to $G L_{2}\left(F_{\wp}\right)$. Consider $\mathbf{K}:=K_{\wp} \times H$, where $K_{\wp}$ is the set of elements of $G L_{2}\left(O_{\wp}\right)$ which are congruent to 1 modulo $\wp$ and $H$ is some open compact subgroup of the restricted product of $\left(D \otimes_{F} F_{\mathfrak{p}}\right)^{\times}$, where $\mathfrak{p}$ runs over all the finite places of $F$, with $\mathfrak{p} \neq \wp$. Then it is well known (see for example [C, Corollary 1.4.1.3]) that for $H$ sufficiently small, the group $G L_{2}(O / \wp)$ acts freely on

$$
S_{\mathbf{K}}(\mathbb{C})=\bar{G}(\mathbb{Q}) \backslash X \times \bar{G}\left(\mathbb{A}_{f}\right) / \mathbf{K} .
$$

We fix such a small $H$. Then the action of $G L_{2}(O / \wp)$ can be described in the following way: $G L_{2}\left(O_{\wp}\right) \hookrightarrow \bar{G}\left(\mathbb{A}_{\mathbb{Q}}\right)$ by $\alpha \mapsto(1, \ldots, \alpha, 1, \ldots, 1), \alpha$ at the $\wp$ component. Using the isomorphism $G L_{2}(O / \wp) \cong G L_{2}\left(O_{\wp}\right) / K_{\wp}$, the action of an element $g \in G L_{2}\left(O_{\wp}\right)$ is given by the right multiplication at the $\wp$-component. 
We fix a continuous representation

$$
\varphi: \Gamma_{E} \longrightarrow G L_{2}(O / \wp) \text {. }
$$

Let $L$ be the finite Galois extension of $E$ defined by $L:=(\overline{\mathbb{Q}})^{\operatorname{Ker}(\varphi)}$.

Let

$$
S^{\prime}=S_{\mathbf{K}} \times_{\operatorname{Spec}(E)} \operatorname{Spec}(L) .
$$

The group $G L_{2}(O / \wp)$ acts on $S_{\mathbf{K}}$. Since $\varphi: \operatorname{Gal}(L / E) \hookrightarrow G L_{2}(O / \wp)$, the group $\operatorname{Gal}(L / E)$ acts on $S_{\mathbf{K}}$. We denote this action of $\operatorname{Gal}(L / E)$ on $S_{\mathbf{K}}$ by $\varphi^{\prime}$. The Galois group $\operatorname{Gal}(L / E)$ has a natural action on $\operatorname{Spec}(L)$ and we can descend via the quotient process $S^{\prime}$ to $S_{\mathbf{K}}^{\prime} / \operatorname{Spec}(E)$ using the diagonal action

$$
\operatorname{Gal}(L / E) \ni \sigma \longrightarrow \varphi^{\prime}(\sigma) \otimes \sigma
$$

on $S^{\prime}$. Thus, we obtain a quasi-projective variety $S_{\mathbf{K}}^{\prime} / \operatorname{Spec}(E)$. This is the twisted quaternionic Shimura variety that we mentioned in the title.

\section{$\S 3 . \quad$ Zeta functions of twisted quaternionic Shimura varieties}

From now on, if $\pi$ is an automorphic representation of $\bar{G}\left(\mathbb{A}_{\mathbb{Q}}\right)$, we denote the automorphic representation of $G L_{2}\left(\mathbb{A}_{F}\right)$, obtained from $\pi$ by JacquetLanglands correspondence (usually denoted $J L(\pi)$ ) by the same symbol $\pi$.

If $\pi$ is an cuspidal automorphic representation of weight 2 of $G L(2) / F$, then there exists $([\mathrm{T}],[\mathrm{C}])$ a $\lambda$-adic representation

$$
\rho_{\pi, \lambda}: \Gamma_{F} \longrightarrow G L_{2}\left(\mathcal{O}_{\lambda}\right) \longleftrightarrow G L_{2}\left(\overline{\mathbb{Q}}_{l}\right),
$$

which satisfies $L(s-1 / 2, \pi)=L\left(s, \rho_{\pi, \lambda}\right)$ and is unramified outside the primes dividing $\mathbf{n} l$. Here $\mathbf{n}$ is the level of $\pi, \mathcal{O}$ is the integer ring of the coefficient field of $\pi$ and $\lambda$ is a prime ideal of $\mathcal{O}$ above some prime number $l$. In order to simplify the notations we denote by $\rho_{\pi}$ the representation $\rho_{\pi, \lambda}$.

We assume that $K=\prod_{v<\infty} K_{v}$ where $K_{v}$ is open compact in $G\left(F_{v}\right)$ and $K_{v}=G L_{2}\left(O_{v}\right)$ for almost all $v$, where $O_{v}$ is the ring of integers of $F_{v}$. Let $\mathbb{H}_{K}$ be the Hecke algebra of convolutions of bi- $K$-invariant $\overline{\mathbb{Q}}_{l}$-valued compactly supported functions on $\bar{G}\left(\mathbb{A}_{f}\right)$. If $\pi=\pi_{\infty} \otimes \pi_{f}$ is an automorphic representation of $\bar{G}\left(\mathbb{A}_{\mathbb{Q}}\right)$, we denote by $\pi_{f}^{K}$ the space of $K$ invariants in $\pi_{f}$. The Hecke algebra $\mathbb{H}_{K}$ acts on $\pi_{f}^{K}$.

In this paper we assume that $D \neq M_{2}(F)$. Then $S_{K}$ is compact.

We have an action of the Hecke algebra $\mathbb{H}_{K}$ and an action of the Galois group $\Gamma_{E}$ on the étale cohomology $H_{e t}^{3}\left(S_{K}, \overline{\mathbb{Q}}_{l}\right)$ and these two actions commute. We say that the representation $\pi$ is cohomological if $H^{3}\left(\mathfrak{g}, K_{\infty}, \pi_{\infty}\right)$ 
$\neq 0$, where $\mathfrak{g}$ is the Lie algebra of $K_{\infty}$ (the cohomology is taken with respect to $\left(\mathfrak{g}, K_{\infty}\right)$-module associated to $\pi_{\infty}$ ). Then we know (see for example [RT, Proposition 1.8]):

Proposition 3.1. The representation of $\Gamma_{E} \times \mathbb{H}_{K}$ on the étale cohomology $H_{e t}^{3}\left(S_{K}, \overline{\mathbb{Q}}_{l}\right)$ is isomorphic to

$$
\bigoplus_{\pi} \rho(\pi) \otimes \pi_{f}^{K}
$$

where $\rho(\pi)$ is a representation of the Galois group $\Gamma_{E}$ of dimension 8. The above sum is over weight 2 cohomological irreducible cuspidal holomorphic automorphic representations $\pi$ of $\bar{G}\left(\mathbb{A}_{\mathbb{Q}}\right)$ and the $\mathbb{H}_{K}$-representations $\pi_{f}^{K}$ are irreducible and mutually inequivalent, i.e. the decomposition is isotypic with respect to the action of $\mathbb{H}_{K}$.

We fix an isomorphism $j: \overline{\mathbb{Q}}_{l} \rightarrow \mathbb{C}$ and define the $L$-function

$$
L^{3}\left(s, S_{K}\right):=\prod_{\pi} \prod_{q} \operatorname{det}\left(1-N q^{-s} j\left(\rho(\pi)\left(\operatorname{Frob}_{q}\right)\right) \mid H_{e t}^{3}\left(S_{K}, \overline{\mathbb{Q}}_{l}\right)^{I_{q}}\right)^{-1},
$$

where Frob $_{q}$ is a geometric Frobenius element at a finite place $q$ of $E$ and $I_{q}$ is a inertia group at $q$. In order to define the local factors at the places of $E$ dividing $l$, one has to use actually the $l^{\prime}$-adic cohomology for some $l^{\prime} \neq l$ and [B, Theorem 3] that gives us the expression of the local factors of the zeta functions of quaternionic Shimura varieties.

We consider the injective limit:

$$
V:=\underset{K}{\lim _{\longrightarrow}} H_{e t}^{3}\left(S_{K}, \overline{\mathbb{Q}}_{l}\right) \cong \underset{K}{\lim _{\pi}} \bigoplus_{\pi} V\left(\pi_{\infty}\right) \otimes_{\overline{\mathbb{Q}}_{l}} \pi_{f}^{K},
$$

where $V\left(\pi_{\infty}\right)$ is the $\overline{\mathbb{Q}}_{l}$-space that corresponds to $\rho(\pi)$ (see Proposition 3.1 for notations).

Using the multiplicity one for $\bar{G}$, we get that the $\pi$-component $V(\pi)$ of $V$ is isomorphic to $\rho(\pi) \otimes \pi_{f}$ as $\Gamma_{E} \times \mathbb{H}$-module. Taking the $\mathbf{K}$-fixed vectors we deduce that $V(\pi)^{\mathbf{K}}$ is isomorphic to $\rho(\pi) \otimes \pi_{f}^{\mathbf{K}}$ as $\Gamma_{E} \times G L_{2}(O / \wp O)$ module. Since the varieties $S_{\mathbf{K}}$ and $S_{\mathbf{K}}^{\prime}$ become isomorphic over $\overline{\mathbb{Q}}$, we have the isomorphism $H_{e t}^{3}\left(S_{\mathbf{K}}, \overline{\mathbb{Q}}_{l}\right) \cong H_{e t}^{3}\left(S_{\mathbf{K}}^{\prime}, \overline{\mathbb{Q}}_{l}\right)$. The actions of $\Gamma_{E}$ on these cohomologies which give the expression of the zeta functions of these varieties are different. If we consider the component $V^{\prime}(\pi)$ that corresponds 
to $\pi$ of $H_{e t}^{3}\left(S_{\mathbf{K}}^{\prime}, \overline{\mathbb{Q}}_{l}\right)$ (see the decomposition of Proposition 3.1), we get that $V^{\prime}(\pi)$ is isomorphic to $\rho(\pi) \otimes\left(\pi_{f}^{\mathbf{K}} \circ \varphi\right)$ as $\Gamma_{E}$-module. Hence we conclude the following result. This is a particular case of Theorem 1.1 from [V]. See [B, Theorem 3], where the local factors of $L^{3}\left(s, S_{\mathbf{K}}\right)$ are computed at all places.

THEOREM 3.2. The L-function $L^{3}\left(s, S_{\mathbf{K}}^{\prime}\right)$ is given by the formula:

$$
L^{3}\left(s, S_{\mathbf{K}}^{\prime}\right)=\prod_{\pi} L\left(s, \rho(\pi) \otimes\left(\pi_{f}^{\mathbf{K}} \circ \varphi\right)\right)
$$

where the product is taken over cohomological irreducible cuspidal holomorphic automorphic representations $\pi$ of $\bar{G}\left(\mathbb{A}_{\mathbb{Q}}\right)$ of weight 2 , such that $\pi_{f}^{\mathbf{K}} \neq 0$.

\section{$\S 4$. Base change}

We know the following result (Theorem 2.2 of $[\mathrm{V}]$ ):

THEOREM 4.1. If $F$ is a totally real field, $\pi$ is a cuspidal automorphic representation of weight 2 of $G L(2) / F$ and $F^{\prime}$ is a solvable extension of a totally real field containing $F$, then there exists a Galois extension $F^{\prime \prime}$ of $\mathbb{Q}$ containing $F^{\prime}$ and there exists a prime $\lambda$ of the field coefficients of $\pi$, such that $\left.\rho_{\pi, \lambda}\right|_{\Gamma_{F^{\prime \prime}}}$ is modular i.e. there exists an automorphic representation $\pi_{1}$ of $G L(2) / F^{\prime \prime}$ and a prime $\beta$ of the field of coefficients of $\pi_{1}$ such that $\left.\rho_{\pi, \lambda}\right|_{\Gamma_{F^{\prime \prime}}} \cong \rho_{\pi_{1}, \beta}$.

In this section we fix an automorphic representation $\pi$ as in Theorem 4.1 and we denote $\omega:=\pi_{f}^{\mathbf{K}} \circ \varphi$. In this paper we assume that the field $L:=$ $\overline{\mathbb{Q}}^{\operatorname{Ker}(\varphi)}$ is a solvable extension of a totally real field. Thus the field $K:=$ $\overline{\mathbb{Q}}^{\operatorname{Ker}(\omega)}$ is a solvable extension of a totally real field.

Let $k$ be a solvable extension of a totally real field which contains $E$. From Theorem 4.1 we deduce that there exists a Galois extension $F^{\prime \prime}$ of $\mathbb{Q}$ containing $F K k$, a prime $\lambda$ of the field coefficients of $\pi$ and an automorphic representation $\pi_{1}$ of $G L(2) / F^{\prime \prime}$ and a prime $\beta$ of the field of coefficients of $\pi_{1}$ such that $\rho_{\pi, \lambda} \mid \Gamma_{F^{\prime \prime}} \cong \rho_{\pi_{1}, \beta}$.

By Brauer's theorem (see [SE, Theorems 16 and 19]), we can find some subfields $F_{i} \subset F^{\prime \prime}$ such that $\operatorname{Gal}\left(F^{\prime \prime} / F_{i}\right)$ are solvable, some characters $\chi_{i}$ : $\operatorname{Gal}\left(F^{\prime \prime} / F_{i}\right) \rightarrow \overline{\mathbb{Q}}^{\times}$and some integers $m_{i}$, such that the representation

$$
\left.\omega\right|_{\Gamma_{k}}: \operatorname{Gal}\left(F^{\prime \prime} / k\right) \longrightarrow \operatorname{Gal}(K k / k) \longrightarrow G L_{N}\left(\overline{\mathbb{Q}}_{l}\right),
$$


can be written as $\left.\omega\right|_{\Gamma_{k}}=\sum_{i=1}^{i=k} m_{i} \operatorname{Ind}_{\Gamma_{F_{i}}}^{\Gamma_{k}} \chi_{i}$ (a virtual sum). Then

$$
\begin{aligned}
& L(s,(\rho(\pi)\left.\otimes \omega)\left.\right|_{\Gamma_{k}}\right)=\prod_{i=1}^{i=k} L\left(s,\left.\rho(\pi)\right|_{\Gamma_{k}} \otimes \operatorname{Ind}_{\Gamma_{F_{i}}}^{\Gamma_{k}} \chi_{i}\right)^{m_{i}} \\
&=\prod_{i=1}^{i=k} L\left(s, \operatorname{Ind}_{\Gamma_{F_{i}}}^{\Gamma_{k}}\left(\left.\rho(\pi)\right|_{\Gamma_{F_{i}}} \otimes \chi_{i}\right)\right)^{m_{i}}=\prod_{i=1}^{i=k} L\left(s,\left.\rho(\pi)\right|_{\Gamma_{F_{i}}} \otimes \chi_{i}\right)^{m_{i}} .
\end{aligned}
$$

If $F \subset F_{i}$, since $\left.\rho_{\pi, \lambda}\right|_{\Gamma_{F^{\prime \prime}}}$ is modular and $\operatorname{Gal}\left(F^{\prime \prime} / F_{i}\right)$ is solvable, from Langlands base change one can deduce that $\left.\rho_{\pi, \lambda}\right|_{\Gamma_{F_{i}}}$ is modular and in this case we denote by $\pi_{i}$ the automorphic representation of $G L(2) / F_{i}$ such that $\left.\rho_{\pi, \lambda}\right|_{\Gamma_{F_{i}}} \cong \rho_{\pi_{i}}$.

\section{$\S 5$. Known results}

It is known that (see for example [HLR, Proposition 4.5.4]):

Proposition 5.1. If $\pi$ is a cuspidal automorphic representation of $G L(2) / F$, where $F$ is a totally real field. Then one of the following two statements holds:

(i) $\left.\rho_{\pi}\right|_{\Gamma_{L}}$ is irreducible for each finite extension $L / F$.

(ii) There exists a quadratic extension $L / F$ and an algebraic Hecke character $\psi$ of $L$ such that $\rho_{\pi} \cong \operatorname{Ind}(\psi)$.

We say that a representation $\rho$ of a group $G$ is dihedral if there exists a normal subgroup $N$ of index 2 in $G$ and a character $\chi: N \rightarrow \mathbb{C}^{\times}$such that $\rho=\operatorname{Ind}_{N}^{G} \chi$

We say that an automorphic representation $\pi$ of $G L(2) / L$ for some number field $L$ is of CM-type if there exists some quadratic Galois character $\eta: I_{L} / L^{\times} \rightarrow \overline{\mathbb{Q}}_{l}^{\times}$, with $\eta \neq 1$ such that $\pi \cong \pi \otimes \eta$. In this case, there exists a quadratic extension $M / F$ and an algebraic Hecke character $\theta$ of $M$ such that $\pi=\pi(\theta)$. If $\pi$ is an automorphic representation of $G L(2) / L$, then $\pi$ is of CM-type if and only if $\rho_{\pi}$ is a dihedral representation.

We know (see [I, Proposition 2.3]):

Proposition 5.2. Let $k$ be a number field and $\pi_{1}, \pi_{2}$ and $\pi_{3}$ be irreducible cuspidal automorphic representations of weight 2 of $G L_{2}\left(\mathbb{A}_{k}\right)$. We denote by $\omega$ the product of the central characters of $\pi_{1}, \pi_{2}$ and $\pi_{3}$. Then, $L\left(s, \pi_{1} \otimes \pi_{2} \otimes \pi_{3}\right)$ can be meromorphically continued to $\mathbb{C}$ and has no zeros 
for $s \geqslant 1$. It is entire if $\omega^{2} \neq 1$ or if $\omega=1$. If $\omega^{2}=1$ and $\omega \neq 1$, then $L\left(s, \pi_{1} \otimes \pi_{2} \otimes \pi_{3}\right)$ has possible poles at $s=0,1$. The function $L\left(s, \pi_{1} \otimes \pi_{2} \otimes \pi_{3}\right)$ satisfies a functional equation

$$
L\left(s, \pi_{1} \otimes \pi_{2} \otimes \pi_{3}\right)=\epsilon\left(s, \pi_{1} \otimes \pi_{2} \otimes \pi_{3}, \sigma\right) L\left(1-s, \tilde{\pi}_{1} \otimes \tilde{\pi}_{2} \otimes \tilde{\pi}_{3}\right) .
$$

We know (see [I, Proposition 2.7]):

Proposition 5.3. Let $k$ be a number field and $\pi_{1}, \pi_{2}$ and $\pi_{3}$ be irreducible cuspidal automorphic representations of weight 2 of $G L_{2}\left(\mathbb{A}_{k}\right)$. We denote by $\omega$ the product of the central characters of $\pi_{1}, \pi_{2}$ and $\pi_{3}$. Assume that $\omega^{2}=1, \omega \neq 1$ and $L\left(s, \pi_{1} \otimes \pi_{2} \otimes \pi_{3}\right)$ has a simple pole at $s=1$. If we denote by $K$ the quadratic extension of $k$ corresponding to $\omega$ by class field theory and by $\theta$ the generator of $\operatorname{Gal}(K / k)$, then there exists quasicharacters $\chi_{1}, \chi_{2}$ and $\chi_{3}$ of $I_{K} / K^{\times}$such that $\pi_{1}=\pi_{1}\left(\chi_{1}\right), \pi_{2}=\pi\left(\chi_{2}\right)$, $\pi_{3}=\pi\left(\chi_{3}\right)$ and $\chi_{1} \chi_{2} \chi_{3}=1$. Also in this case we have

$$
L\left(s, \pi_{1} \otimes \pi_{2} \otimes \pi_{3}\right)=\zeta_{K}(s) L_{K}\left(s, \chi_{1}^{-1} \chi_{1}^{\theta}\right) L_{K}\left(s, \chi_{2}^{-1} \chi_{2}^{\theta}\right) L_{K}\left(s, \chi_{3}^{-1} \chi_{3}^{\theta}\right) .
$$

Let $k$ be a number field and $k^{\prime}$ a cubic extension of $k$. Let $H$ be the algebraic group defined over $k$ given by

$$
H=\left\{g \in \operatorname{Res}_{k^{\prime} / k} G L_{2} \mid \operatorname{det} g \in k^{\times}\right\} .
$$

Let $\Pi$ be an irreducible cuspidal automorphic representation of $G L_{2}\left(\mathbb{A}_{k^{\prime}}\right)$. The $L$-group of $H$ is a semi-direct product of $\Gamma_{k}$ and $G L_{2}(\mathbb{C}) \times G L_{2}(\mathbb{C}) \times$ $G L_{2}(\mathbb{C})$ and $\Gamma_{k}$ acts by permuting the three factors $G L_{2}(\mathbb{C})$. We consider the representation $\sigma$ of the $L$-group of $H$ such that the restriction of $\sigma$ to $G L_{2}(\mathbb{C}) \times G L_{2}(\mathbb{C}) \times G L_{2}(\mathbb{C})$ is $\sigma_{2} \otimes \sigma_{2} \otimes \sigma_{2}$, where $\sigma_{2}$ is the standard 2-dimensional representation of $G L_{2}(\mathbb{C})$, and the restriction of $\sigma$ to $\Gamma_{k}$ permutes the three factors. We consider the $L$-function $L(s, \Pi, \sigma)$ given by

$$
L(s, \Pi, \sigma):=\prod_{v} L\left(s, \Pi_{v}, \sigma\right),
$$

where $v$ runs over the finite places of $k$ and if $\Pi$ is unramified at $v$, then the local factor $L\left(s, \Pi_{v}, \sigma\right)$ is given by

$$
L\left(s, \Pi_{v}, \sigma\right):=\operatorname{det}\left(1_{8}-\sigma\left(\operatorname{Frob}_{v}, g_{v}\right) N v^{-s}\right)^{-1},
$$

where $\operatorname{Frob}_{v}$ is a geometric Frobenius at $v$ and $g_{v}$ is the Langlands class of $\Pi_{v}$.

Then, we know (see [I, Proposition 2.3]): 
Proposition 5.4. $L(s, \Pi, \sigma)$ can be meromorphically continued to $\mathbb{C}$ and has no zeros for $s \geqslant 1$. Let $\Omega$ the cental character of $\Pi$ and $\omega$ the restriction of $\Omega$ to the center $Z\left(\mathbb{A}_{k}\right)$. The function $L(s, \Pi, \sigma)$ is entire if $\omega^{2}$ is not a principal quasi-character. If $\omega^{2}=1$, then $L(s, \Pi, \sigma)$ has possible poles at $s=0,1$. The function $L(s, \Pi, \sigma)$ satisfies a functional equation

$$
L(s, \Pi, \sigma)=\epsilon(s, \Pi, \sigma) L(1-s, \tilde{\Pi}, \sigma) .
$$

We know (see [I, Proposition 2.6]):

Proposition 5.5. Let $k$ be a number field and $k^{\prime}$ a cubic extension of $k$. Assume that $L(s, \Pi, \sigma)$ has a pole at $s=1$. Then $\omega^{2}=1, \omega \neq 1$. Let $K$ be the quadratic extension of $k$ corresponding to $\omega$ by class field theory. Let $\theta$ be the non-trivial element of $\operatorname{Gal}\left(k^{\prime} K / k^{\prime}\right)$. Then there exists a quasicharacter $\chi$ of $I_{k^{\prime} K} / k^{\prime} K^{\times}$such that $\Pi=\pi(\chi)$ and $\left.\chi\right|_{I_{K}}=1$. Moreover, the function $L(s, \Pi, \sigma)$ is given by

$$
L(s, \pi(\chi), \sigma)=\zeta_{K}(s) L_{k^{\prime} K}\left(s, \chi^{-1} \chi^{\theta}\right) .
$$

Let $k$ be a number field and $k^{\prime}$ be a quadratic extension of $k$. Let $H$ be the algebraic group defined over $k$ given by

$$
H=\left\{\left(g^{(1)}, g^{(2)}\right) \in G L_{2} \times \operatorname{Res}_{k^{\prime} / k} G L_{2} \mid \operatorname{det} g^{(1)}=\operatorname{det} g^{(2)}\right\} .
$$

Let $\Pi$ be in irreducible cuspidal automorphic representation of $G L_{2}\left(\mathbb{A}_{k} \otimes\right.$ $\left(k \oplus k^{\prime}\right)$ ), i.e. $\Pi=\pi_{1} \otimes \pi_{2}$, where $\pi_{1}$ and $\pi_{2}$ are irreducible representations of $G L_{2}\left(\mathbb{A}_{k}\right)$ and $G L_{2}\left(\mathbb{A}_{k^{\prime}}\right)$ respectively.

The $L$-group of $H$ is also the semi-direct product of $\Gamma_{k}$ and $G L_{2}(\mathbb{C}) \times$ $G L_{2}(\mathbb{C}) \times G L_{2}(\mathbb{C})$ and $\Gamma_{k}$ acts by permuting the three factors $G L_{2}(\mathbb{C})$. Then Proposition 5.4 remains true for $L(s, \Pi, \sigma)$ and we know (see [I, Proposition 2.3]):

Proposition 5.6. Let $k$ be a number field and $k^{\prime}$ be a quadratic extension of $k$. Assume that $L(s, \Pi, \sigma)$ has a pole at $s=1$. Then $\omega^{2}=1, \omega \neq 1$. Let $K$ be the quadratic extension of $k$ corresponding to $\omega$ by class field theory. Let $\theta$ be the non-trivial element of $\operatorname{Gal}\left(k^{\prime} K / k^{\prime}\right)$. Then there exists a quasi-character $\chi$ of $I_{k^{\prime} K} / k^{\prime} K$ such that $\pi_{1}=\pi\left(\left.\chi^{-1}\right|_{I_{K}}\right)$, and $\pi_{2}=\pi(\chi)$. Moreover, the L-function is equal to

$$
\zeta_{K}(s) L_{K}\left(s,\left.\left(\chi^{-1} \chi^{\theta}\right)\right|_{I_{K}}\right) L_{k^{\prime} K}\left(s, \chi^{-1} \chi^{\theta}\right) .
$$




\section{$\S 6$. Poles of $L$-functions of twisted quaternionic Shimura varieties}

Assume that $k$ is a solvable extension of a totally real field which contains $E$ and $\pi$ is a cuspidal automorphic representation of $G L(2) / F$ that appears in Theorem 3.2.

We recall that in Section 4 we denoted $\omega:=\pi_{f}^{\mathbf{K}} \circ \varphi$ and we have assumed that the field $L:=\overline{\mathbb{Q}}^{\operatorname{Ker} \varphi}$ is a solvable extension of a totally real field.

We denote by $\rho(\pi)^{s s}$ the semisimplification of $\rho(\pi)$. Since at all but a finite number of finite places of $E$, the representations $\left.\rho(\pi)\right|_{\Gamma_{F_{i}}}$ and $\left.\rho(\pi)^{s s}\right|_{\Gamma_{F_{i}}}$ yield the same local $L$-factors, and because from the Weight-Monodromy conjecture that is true for $\rho(\pi)$ and $\rho(\pi)^{s s}$ (see [B, Theorem 2]), we know that the poles of the $L$-factors corresponding to $\rho(\pi)$ and $\rho(\pi)^{s s}$ are on the line $\operatorname{Re}(s)=3 / 2$ (see [B, Theorem 2]), we deduce that the order of the pole at some $s$ with $\operatorname{Re}(s) \geqslant 5 / 2$ of $L\left(s,\left.(\rho(\pi) \otimes \omega)\right|_{\Gamma_{K}}\right)=\prod_{i=1}^{i=k} L\left(s,\left.\rho(\pi)\right|_{\Gamma_{F_{i}}} \otimes\right.$ $\left.\chi_{i}\right)^{m_{i}}$ is equal to the order of the pole at $s$ of $L\left(s,\left.\left(\rho(\pi)^{s s} \otimes \omega\right)\right|_{\Gamma_{k}}\right)^{i}=$ $\prod_{i=1}^{i=k} L\left(s,\left.\rho(\pi)^{s s}\right|_{\Gamma_{F_{i}}} \otimes \chi_{i}\right)^{m_{i}}$.

In this paper we show the following result:

THEOREM 6.1. If $k$ is a solvable extension of a totally real field containing $E$, and $K:=\overline{\mathbb{Q}}^{\operatorname{Ker}(\omega)}$ is a solvable extension of a totally real field, then the function $L\left(s,\left.\left(\rho(\pi)^{s s} \otimes \omega\right)\right|_{\Gamma_{k}}\right)$ has a meromorphic continuation and satisfies a functional equation $s \leftrightarrow 4-s$ and it has no zeros and is holomorphic in the half plane $\operatorname{Re}(s) \geqslant 5 / 2$.

Since, $L\left(s,\left.\left(\rho(\pi)^{s s} \otimes \omega\right)\right|_{\Gamma_{k}}\right)=\prod_{i=1}^{i=k} L\left(s,\left.\rho(\pi)^{s s}\right|_{\Gamma_{F_{i}}} \otimes \chi_{i}\right)^{m_{i}}$, in order to prove Theorem 6.1, it is sufficient to show that $L\left(s,\left.\rho(\pi)^{s s}\right|_{\Gamma_{F_{i}}} \otimes \chi_{i}\right)$ is holomorphic, satisfies a functional equation $s \leftrightarrow 4-s$ and does not vanish in the half plane $\operatorname{Re}(s) \geqslant 5 / 2$.

We describe now the representation $\rho^{s s}(\pi)$ (see [BR, $\left.\S 7.2\right]$ ). Let $G$ be a group and $K$ and $H$ be two subgroups of $G$. We consider a representation

$$
\tau: H \longrightarrow G L(W)
$$

and a double coset $H \sigma K$ such that $d(\sigma)=|H \backslash H \sigma K|<\infty$. We define a representation $\tau_{H \sigma K}$ of $K$ on the $d(\sigma)$-fold tensor product $W^{\otimes d(\sigma)}$. Consider the representatives $\left\{\sigma_{1}, \ldots, \sigma_{d(\sigma)}\right\}$ such that $H \sigma K=\bigcup H \sigma_{j}$. If $\gamma \in K$, then there exists $\xi_{j} \in H$ and an index $\gamma(j)$ such that

$$
\sigma_{j} \gamma=\xi_{j} \sigma_{\gamma(j)}
$$


We define the representation:

$$
\tau_{H \sigma K}(\gamma)\left(\omega_{1} \otimes \cdots \otimes \omega_{d(\sigma)}\right)=\tau\left(\xi_{1}\right) \omega_{\gamma^{-1}(1)} \otimes \cdots \otimes \tau\left(\xi_{d(\sigma)}\right) \omega_{\gamma^{-1}(d(\sigma))}
$$

One can prove easily that the equivalence class of $\tau_{H \sigma K}$ is independent of the choice of the representatives $\sigma_{1}, \ldots, \sigma_{d(\sigma)}$.

Let $S_{\infty}-S_{\infty}^{\prime}=\left\{\tau_{1}, \tau_{2}, \tau_{3}\right\}$, and $S:=\bigcup \Gamma_{F} \tau_{i}$. We write $S$ as a disjoint union of double cosets

$$
S=\bigcup_{j=1}^{k} \Gamma_{F} \tau_{j} \Gamma_{E}
$$

and we denote by $\rho_{j}$ the representation of $\Gamma_{E}$ defined by $\rho_{\pi, \lambda}$ and the double $\operatorname{coset} \Gamma_{F} \tau_{j} \Gamma_{E}$. Then our representation $\rho^{s s}(\pi)$ is isomorphic to $\rho_{1} \otimes \cdots \otimes \rho_{k}$.

We assume for simplicity that $S_{\infty}-S_{\infty}^{\prime}=\left\{1, \tau_{1}, \tau_{2}\right\}$, where 1 is the trivial embedding of $F$ in $\overline{\mathbb{Q}}$. We denote by the same symbol $\tau_{i}$ the extension of $\tau_{i}$ to $\overline{\mathbb{Q}}$. Consider

$$
S=\Gamma_{F} \cup \Gamma_{F} \tau_{1} \cup \Gamma_{F} \tau_{2} .
$$

The stabilizer of $S$ is $\Gamma_{E}$. It is easy to see that we have

$$
\begin{aligned}
\Gamma_{E}= & \left(\Gamma_{F} \cap \tau_{1}^{-1} \Gamma_{F} \tau_{1} \cap \tau_{2}^{-1} \Gamma_{F} \tau_{2}\right) \cup\left(\Gamma_{F} \cap \tau_{1}^{-1} \Gamma_{F} \tau_{2} \cap \tau_{2}^{-1} \Gamma_{F} \tau_{1}\right) \\
& \cup\left(\Gamma_{F} \tau_{2} \cap \tau_{1}^{-1} \Gamma_{F} \tau_{1} \cap \tau_{2}^{-1} \Gamma_{F}\right) \cup\left(\Gamma_{F} \tau_{1} \cap \tau_{1}^{-1} \Gamma_{F} \cap \tau_{2}^{-1} \Gamma_{F} \tau_{2}\right) \\
& \cup\left(\Gamma_{F} \tau_{1} \cap \tau_{1}^{-1} \Gamma_{F} \tau_{2} \cap \tau_{2}^{-1} \Gamma_{F}\right) \cup\left(\Gamma_{F} \tau_{2} \cap \tau_{2}^{-1} \Gamma_{F} \tau_{1} \cap \tau_{1}^{-1} \Gamma_{F}\right)
\end{aligned}
$$

We distinguish several cases:

i) $F \subset E$. Then,

$$
\Gamma_{E}=\left(\Gamma_{F} \cap \tau_{1}^{-1} \Gamma_{F} \tau_{1} \cap \tau_{2}^{-1} \Gamma_{F} \tau_{2}\right) \cup\left(\Gamma_{F} \cap \tau_{1}^{-1} \Gamma_{F} \tau_{2} \cap \tau_{2}^{-1} \Gamma_{F} \tau_{1}\right) .
$$

We consider two subcases:

a) $\Gamma_{F} \cap \tau_{1}^{-1} \Gamma_{F} \tau_{2} \cap \tau_{2}^{-1} \Gamma_{F} \tau_{1}=\varnothing$.

If $\pi$ is cuspidal automorphic representation, we denote for simplicity $\rho_{\pi}:=\rho_{\pi, \lambda}$. Then we obtain

$$
\left.\left.\left.\rho(\pi)^{s s} \cong \rho_{\pi}\right|_{\Gamma_{E}} \otimes \rho_{\pi}\right|_{\Gamma_{E}} ^{\tau_{1}} \otimes \rho_{\pi}\right|_{\Gamma_{E}} ^{\tau_{2}}
$$

where

$$
\left.\rho_{\pi}\right|_{\Gamma_{E}} ^{\tau_{i}}(\gamma)=\rho_{\pi}\left(\tau_{i} \gamma \tau_{i}^{-1}\right)
$$


b) $\Gamma_{F} \cap \tau_{1}^{-1} \Gamma_{F} \tau_{2} \cap \tau_{2}^{-1} \Gamma_{F} \tau_{1} \neq \varnothing$. Define $\Gamma_{E_{1}}:=\Gamma_{F} \cap \tau_{1}^{-1} \Gamma_{F} \tau_{1} \cap \tau_{2}^{-1} \Gamma_{F} \tau_{2}$. It is easy to see that $\left[E_{1}: E\right]=2, F \subset E$ and that

$$
\left.\rho(\pi)^{s s} \cong \rho_{\pi}\right|_{\Gamma_{E}} \otimes \rho(\pi)^{\prime},
$$

where $\rho(\pi)^{\prime}$ is a subrepresentation of

$$
\operatorname{Ind}_{\Gamma_{E_{1}}}^{\Gamma_{E}}\left(\left.\left.\rho_{\pi}\right|_{\Gamma_{E_{1}}} ^{\tau_{1}} \otimes \rho_{\pi}\right|_{\Gamma_{E_{1}}} ^{\tau_{2}}\right),
$$

which verifies

$$
\left.\rho(\pi)^{\prime}\right|_{\Gamma_{E_{1}}}=\left.\left.\rho_{\pi}\right|_{\Gamma_{E_{1}}} ^{\tau_{1}} \otimes \rho_{\pi}\right|_{\Gamma_{E_{1}}} ^{\tau_{2}}
$$

We distinguish two subcases:

1) $E_{1} \subseteq F_{i}$. Then

$$
\left.\left.\left.\left.\rho(\pi)^{s s}\right|_{\Gamma_{F_{i}}} \cong \rho_{\pi}\right|_{\Gamma_{F_{i}}} \otimes \rho_{\pi}\right|_{\Gamma_{F_{i}}} ^{\tau_{1}} \otimes \rho_{\pi}\right|_{\Gamma_{F_{i}}} ^{\tau_{2}}
$$

2) $E_{1} \nsubseteq F_{i}$. Since $\left.\rho_{\pi, \lambda}\right|_{\Gamma_{F_{i}}}$ is $E_{1} F_{i}$ is a quadratic extension of $F_{i}$, we obtain that $\left.\rho_{\pi}\right|_{\Gamma_{E_{1} F_{i}}}$ is modular and thus there exists an automorphic representation $\pi_{i}^{\prime}$ of $G L(2) / E_{1} F_{i}$ such that $\left.\rho_{\pi, \lambda}\right|_{\Gamma_{E_{1} F_{i}}} \cong \rho_{\pi_{i}^{\prime}}$.

One can check easily that

$$
L\left(s,\left.\rho(\pi)^{s s}\right|_{F_{i}} \otimes \chi_{i}\right)=L\left(s-3 / 2,\left(\pi_{i} \otimes \chi_{i}\right) \otimes \pi_{i}^{\prime \tau_{1}}, \sigma\right),
$$

where $\pi_{i}$ is the base change of $\pi$ to $G L(2) / F_{i}$ defined at the end of Section 4 .

We remark that this equality is true even when $\pi_{i}$ is reducible, i.e. when (by Proposition 5.1) the representation $\pi$ is of CM-type, $\pi=\pi(\theta)$, where $\theta$ is an algebraic character of $M$ for some quadratic extension $M / F$ and $F_{i}$ contains $M$. Also the results in Theorems 5.2, 5.3, 5.4, 5.5 and 5.6 about the functional equation and the existence the poles of the $L$-functions remain true for such reducible representations $\pi_{i}$ (see below Section 6.2).

ii) $\tau_{1}(F) \subset E$. This case is similar to case i), and some subcases overlap.

iii) $\tau_{2}(F) \subset E$. This case is also similar to case i), and some subcases overlap.

iv) $\Gamma_{F} \tau_{1} \cap \tau_{1}^{-1} \Gamma_{F} \tau_{2} \cap \tau_{2}^{-1} \Gamma_{F} \neq \varnothing$. It is easy to see (after replacing $\tau_{1}$ and $\tau_{2}$ by other representatives of $\Gamma_{F} \tau_{1}$ and $\Gamma \tau_{2}$ if necessary) that $\tau_{1}^{3}=1$ and $\tau_{1}^{2}=\tau_{2}$.

We consider two cases: 
a) $\Gamma_{F} \cap \tau_{1}^{-1} \Gamma_{F} \tau_{2} \cap \tau_{2}^{-1} \Gamma_{F} \tau_{1}=\varnothing$. Then it is easy to see that, $[F: E]=3$, $F$ is Galois over $E$ and $\tau_{1}$ is the non-trivial automorphism of $F$ over $E$. We get that $\rho(\pi)^{s s}$ is a subrepresentation of

$$
\operatorname{Ind}_{\Gamma_{F}}^{\Gamma_{E}}\left(\rho_{\pi} \otimes \rho_{\pi}^{\tau_{1}} \otimes \rho_{\pi}^{\tau_{1}^{2}}\right)
$$

which verifies

$$
\left.\rho(\pi)^{s s}\right|_{\Gamma_{F}} \cong \rho_{\pi} \otimes \rho_{\pi}^{\tau_{1}} \otimes \rho_{\pi}^{\tau_{1}^{2}} .
$$

b) $\Gamma_{F} \cap \tau_{1}^{-1} \Gamma_{F} \tau_{2} \cap \tau_{2}^{-1} \Gamma_{F} \tau_{1} \neq \emptyset$. Let $\Gamma_{E_{1}}:=\Gamma_{F} \cap \tau_{1}^{-1} \Gamma_{F} \tau_{1} \cap \tau_{2}^{-1} \Gamma_{F} \tau_{2}$. Then it is easy to see that $[F: E]=3, F$ is not Galois over $E,\left[E_{1}: F\right]=2$ and $E_{1}$ is Galois over $E$.

We distinguish several subcases:

1) $E_{1} \subseteq F_{i}$. Then we get

$$
\left.\left.\left.\left.\rho(\pi)^{s s}\right|_{\Gamma_{F_{i}}} \cong \rho_{\pi}\right|_{\Gamma_{F_{i}}} \otimes \rho_{\pi}\right|_{\Gamma_{F_{i}}} ^{\tau_{1}} \otimes \rho_{\pi}\right|_{\Gamma_{F_{i}}} ^{\tau_{1}^{2}} .
$$

2) $F \nsubseteq F_{i}, \tau_{1}(F) \nsubseteq F_{i}$ and $\tau_{1}^{2}(F) \nsubseteq F_{i}$. Since $\left.\rho_{\pi, \lambda}\right|_{\Gamma_{F^{\prime \prime}}}$ is modular and $F^{\prime \prime}$ is a solvable extension of $F F_{i}$, we obtain that $\left.\rho_{\pi}\right|_{\Gamma_{F F_{i}}}$ is modular and thus there exists a cuspidal automorphic representation $\pi_{i}^{\prime}$ of $G L(2) / F F_{i}$ such that $\left.\rho_{\pi, \lambda}\right|_{\Gamma_{F F_{i}}} \cong \rho_{\pi_{i}^{\prime}}$. Then, $F F_{i}$ is an extension of degree 3 of $F_{i}$ and the element $\tau_{1}$ can be regarded as the nontrivial embedding of $F F_{i}$ over $F_{i}$.

One could check easily that

$$
L\left(s,\left.\rho(\pi)^{s s}\right|_{\Gamma_{F_{i}}} \otimes \chi_{i}\right)=L\left(s-3 / 2, \pi_{i}^{\prime} \otimes \chi_{i}^{\prime}, \sigma\right),
$$

where $\chi_{i}^{\prime}=\left.\chi_{i}\right|_{\Gamma_{F F_{i}}}$.

3) $F \subseteq F_{i}$, but $E_{1} \nsubseteq F_{i}$. Then

$$
\left.\left.\rho(\pi)^{s s}\right|_{\Gamma_{F_{i}}} \cong \rho_{\pi}\right|_{\Gamma_{F_{i}}} \otimes \rho(\pi)_{i}^{\prime},
$$

where $\rho(\pi)_{i}^{\prime}$ is a subrepresentation of

$$
\operatorname{Ind}_{\Gamma_{F_{i} E_{1}}}^{\Gamma_{F_{i}}}\left(\left.\left.\rho_{\pi}\right|_{\Gamma_{F_{i} E_{1}}} ^{\tau_{1}} \otimes \rho_{\pi}\right|_{\Gamma_{F_{i} E_{1}}} ^{\tau_{1}^{2}}\right)
$$

which verifies

$$
\left.\rho(\pi)_{i}^{\prime}\right|_{\Gamma_{F_{i} E_{1}}}=\left.\left.\rho_{\pi}\right|_{\Gamma_{F_{i} E_{1}}} ^{\tau_{1}} \otimes \rho_{\pi}\right|_{\Gamma_{F_{i} E_{1}}^{2}} ^{\tau_{1}^{2}}
$$

4) $\tau_{1}(F) \subseteq F_{i}$, but $E_{1} \nsubseteq F_{i}$. This case is similar to the case 3 ).

5) $\tau_{1}^{2}(F) \subseteq F_{i}$, but $E_{1} \nsubseteq \subseteq F_{i}$. This case is also similar to the case 3 ). 


\subsection{Non-CM case}

In this section we assume that our automorphic representation $\pi$ of $G L(2) / F$ is non-CM.

In the case i) described above, we have two subcases:

a) $F \subseteq E$ and

$$
\left.\left.\left.\rho(\pi)^{s s} \cong \rho_{\pi}\right|_{\Gamma_{E}} \otimes \rho_{\pi}\right|_{\Gamma_{E}} ^{\tau_{1}} \otimes \rho_{\pi}\right|_{\Gamma_{E}} ^{\tau_{2}}
$$

The representation $\pi$ is non-CM and from Proposition 5.1, we get that the representation $\pi_{i}$ is non- $\mathrm{CM}$. Then

$$
\begin{aligned}
L\left(s,\left.\rho(\pi)^{s s}\right|_{\Gamma_{F_{i}}} \otimes \chi_{i}\right) & =L\left(s, \rho_{\pi_{i}} \otimes \rho_{\pi_{i}}^{\tau_{1}} \otimes \rho_{\pi_{i}}^{\tau_{2}} \otimes \chi_{i}\right) \\
& =L\left(s-3 / 2, \pi_{i} \otimes \pi_{i}^{\tau_{1}} \otimes \pi_{i}^{\tau_{2}} \otimes \chi_{i}\right) .
\end{aligned}
$$

Since the representation $\pi_{i}$ is cuspidal non-CM, from Propositions 5.2 and 5.3 , we know that the $L$-function $L\left(s-3 / 2, \pi_{i} \otimes \pi_{i}^{\tau_{1}} \otimes \pi_{i}^{\tau_{2}} \otimes \chi_{i}\right)$ has no poles and does not vanish for $\operatorname{Re}(s) \geqslant 5 / 2$ and satisfies a functional equation.

b) As we said above we consider two subcases:

1) $E_{1} \subseteq F_{i}$. Then

$$
\left.\left.\left.\left.\rho(\pi)^{s s}\right|_{\Gamma_{F_{i}}} \cong \rho_{\pi}\right|_{\Gamma_{F_{i}}} \otimes \rho_{\pi}\right|_{\Gamma_{F_{i}}} ^{\tau_{1}} \otimes \rho_{\pi}\right|_{\Gamma_{F_{i}}} ^{\tau_{2}},
$$

and we are in the same situation as in the case a).

2) $E_{1} \not \subseteq F_{i}$. Then

$$
L\left(s,\left.\rho(\pi)^{s s}\right|_{\Gamma_{F_{i}}} \otimes \chi_{i}\right)=L\left(s-3 / 2,\left(\pi_{i} \otimes \chi_{i}\right) \otimes \pi_{i}^{\prime \tau_{1}}, \sigma\right),
$$

where $\pi_{i}^{\prime}$ is, as above, the cuspidal automorphic representation of $G L(2) / F F_{i}$ such that $\left.\rho_{\pi, \lambda}\right|_{\Gamma_{F F_{i}}} \cong \rho_{\pi_{i}^{\prime}}$. Because $\pi$ is non-CM, from Propositions 5.1, we get that $\pi_{i}^{\prime}$ is non-CM. Since the representation $\pi_{i}^{\prime}$ is cuspidal non-CM, from Propositions 5.4 and 5.6, we know that the $L$-function $L\left(s-3 / 2,\left(\pi_{i} \otimes \chi_{i}\right) \otimes\right.$ $\left.\pi_{i}^{\prime \tau_{1}}, \sigma\right)$ has no poles and does not vanish for $\operatorname{Re}(s) \geqslant 5 / 2$ and satisfies a functional equation.

The cases ii) and iii) are similar to the case i).

In the case iv), a), we know that $[F: E]=3, F$ is Galois over $E, \tau_{1}$ is the non-trivial automorphism of $F$ over $E$ and $\rho(\pi)^{s s}$ is a subrepresentation of

$$
\operatorname{Ind}_{\Gamma_{F}}^{\Gamma_{E}}\left(\rho_{\pi} \otimes \rho_{\pi}^{\tau_{1}} \otimes \rho_{\pi}^{\tau_{1}^{2}}\right)
$$


which verifies

$$
\left.\rho(\pi)^{s s}\right|_{\Gamma_{F}} \cong \rho_{\pi} \otimes \rho_{\pi}^{\tau_{1}} \otimes \rho_{\pi}^{\tau_{1}^{2}}
$$

We distinguish two subcases:

1) $F \subseteq F_{i}$. Then

$$
\left.\left.\left.\left.\rho(\pi)^{s s}\right|_{\Gamma_{F_{i}}} \cong \rho_{\pi}\right|_{\Gamma_{F_{i}}} \otimes \rho_{\pi}\right|_{\Gamma_{F_{i}}} ^{\tau_{1}} \otimes \rho_{\pi}\right|_{\Gamma_{F_{i}}} ^{\tau_{1}^{2}},
$$

and we are in the same situation as in the case i), a).

2) $F \nsubseteq F_{i}$. Then

$$
L\left(s,\left.\rho(\pi)^{s s}\right|_{\Gamma_{F_{i}}} \otimes \chi_{i}\right)=L\left(s-3 / 2, \pi_{i}^{\prime} \otimes \chi_{i}^{\prime}, \sigma\right) .
$$

Because $\pi$ is non-CM, from Propositions 5.1, we get that $\pi_{i}^{\prime}$ is nonCM. Since the representation $\pi_{i}^{\prime}$ is cuspidal non-CM, from Propositions 5.4 and 5.5, we know that the function $L\left(s-3 / 2, \pi_{i}^{\prime} \otimes \chi_{i}^{\prime}, \sigma\right)$ is holomorphic and does not vanish for $\operatorname{Re}(s) \geqslant 5 / 2$ and satisfies a functional equation.

The case iv), b), 1) is similar to the case i), a); the case iv), b), 2) is similar to the case i), b), 2); the cases iv), b), 3) and iv), b), 4) and iv), b), 5) are similar to the case i), b).

\subsection{CM case}

We assume that our representation $\pi$ is cuspidal of CM-type. From the fact that our representation $\pi$ is cohomological, we deduce there exists a quadratic CM-extension $M / F$ and an algebraic Hecke character $\theta$ of $M$ such that $\pi=\pi(\theta)$. We denote by $\alpha$ the non-trivial element of $\operatorname{Gal}(M / F)$.

In the case i), a) from Section 6 we know that:

$$
\left.\left.\left.\rho(\pi)^{s s} \cong \rho_{\pi}\right|_{\Gamma_{E}} \otimes \rho_{\pi}^{\tau_{1}}\right|_{\Gamma_{E}} \otimes \rho_{\pi}^{\tau_{2}}\right|_{\Gamma_{E}}
$$

Thus

$$
\begin{aligned}
L\left(s,\left.\rho(\pi)^{s s}\right|_{\Gamma_{F_{i}}} \otimes \chi_{i}\right) & =L\left(s, \rho_{\pi_{i}} \otimes \rho_{\pi_{i}}^{\tau_{1}} \otimes \rho_{\pi_{i}}^{\tau_{2}} \otimes \chi_{i}\right) \\
& =L\left(s-3 / 2, \pi_{i} \otimes \pi_{i}^{\tau_{1}} \otimes \pi_{i}^{\tau_{2}} \otimes \chi_{i}\right) .
\end{aligned}
$$

From Propositions 5.2 and 5.3, we get that the order of the pole of $L\left(s-3 / 2, \pi_{i} \otimes \pi_{i}^{\tau_{1}} \otimes \pi_{i}^{\tau_{2}} \otimes \chi_{i}\right)$ at $s=5 / 2$ is 1 if $\theta_{i} \theta_{i}^{\tau_{1}} \theta_{i}^{\tau_{2}} \chi_{i}^{\prime \prime}=1$ or $\theta_{i}^{\alpha} \theta_{i}^{\tau_{1}} \theta_{i}^{\tau_{2}} \chi_{i}^{\prime \prime}=$ 1 or $\theta_{i} \theta_{i}^{\alpha \tau_{1}} \theta_{i}^{\tau_{2}} \chi_{i}^{\prime \prime}=1$ or $\theta_{i} \theta_{i}^{\tau_{1}} \theta_{i}^{\alpha \tau_{2}} \chi_{i}^{\prime \prime}=1$, where $\theta_{i}=\left.\theta\right|_{\Gamma_{F_{i} M}}, \chi_{i}^{\prime \prime}=\left.\chi_{i}\right|_{\Gamma_{F_{i} M}}$. Otherwise the function $L\left(s-3 / 2, \pi_{i} \otimes \pi_{i}^{\tau_{1}} \otimes \pi_{i}^{\tau_{2}} \otimes \chi_{i}\right)$ is holomorphic at 
$s=5 / 2$. Also, the function $L\left(s-3 / 2, \pi_{i} \otimes \pi_{i}^{\tau_{1}} \otimes \pi_{i}^{\tau_{2}} \otimes \chi_{i}\right)$ is non-zero for $\operatorname{Re}(s) \geqslant 5 / 2$ and satisfies a functional equation.

But since the CM-weight of $\theta$ is $\left(\frac{1}{2}, \ldots, \frac{1}{2}, \frac{-1}{2}, \ldots, \frac{-1}{2}\right)$, where the number of $\frac{1}{2}$ 's is equal to $[F: \mathbb{Q}]$ and the number of $\frac{-1}{2}$ 's is equal to $[F: \mathbb{Q}]$, we see easily that it is impossible any of the characters $\theta_{i} \theta_{i}^{\tau_{1}} \theta_{i}^{\tau_{2}} \chi_{i}^{\prime \prime}, \theta_{i}^{\alpha} \theta_{i}^{\tau_{1}} \theta_{i}^{\tau_{2}} \chi_{i}^{\prime \prime}$, $\theta_{i} \theta_{i}^{\alpha \tau_{1}} \theta_{i}^{\tau_{2}} \chi_{i}^{\prime \prime}$ or $\theta_{i} \theta_{i}^{\tau_{1}} \theta_{i}^{\alpha \tau_{2}} \chi_{i}^{\prime \prime}$ to have parallel weight $(0, \ldots, 0)$ and thus all these 4 characters are different from 1 and we obtain that the function $L\left(s-3 / 2, \pi_{i} \otimes \pi_{i}^{\tau_{1}} \otimes \pi_{i}^{\tau_{2}} \otimes \chi_{i}\right)$ is holomorphic at $s=5 / 2$.

In the case i), b) we distinguish two subcases:

1) $E_{1} \subseteq F_{i}$. Then

$$
\left.\left.\left.\left.\rho(\pi)^{s s}\right|_{\Gamma_{F_{i}}} \cong \rho_{\pi}\right|_{\Gamma_{F_{i}}} \otimes \rho_{\pi}\right|_{\Gamma_{F_{i}}} ^{\tau_{1}} \otimes \rho_{\pi}\right|_{\Gamma_{F_{i}}} ^{\tau_{2}},
$$

and we are in the same situation as in the case i), a).

2) $E_{1} \nsubseteq F_{i}$. Then

$$
L\left(s,\left.\rho(\pi)^{s s}\right|_{\Gamma_{F_{i}}} \otimes \chi_{i}\right)=L\left(s-3 / 2,\left(\pi_{i} \otimes \chi_{i}\right) \otimes \pi_{i}^{\prime \tau_{1}}, \sigma\right) .
$$

From Propositions 5.4 and 5.6, we get that the order of the pole of $L\left(s-3 / 2,\left(\pi_{i} \otimes \chi_{i}\right) \otimes \pi_{i}^{\prime \tau_{1}}, \sigma\right)$ at $s=5 / 2$ is equal to 1 if $\theta_{i}^{\prime \tau_{1}} \mid I_{M F_{i}}=\left(\theta_{i} \chi_{i}^{\prime \prime}\right)^{-1}$ or $\theta_{i}^{\prime \tau_{1}} \mid I_{M F_{i}}=\left(\theta_{i}^{\alpha} \chi_{i}^{\prime \prime}\right)^{-1}$, where $\theta_{i}=\left.\theta\right|_{\Gamma_{M F_{i}}}, \chi_{i}^{\prime \prime}=\left.\chi_{i}\right|_{\Gamma_{M F_{i}}}$ and $\theta_{i}^{\prime}=\left.\theta\right|_{\Gamma_{E_{1} M F_{i}}}$. Otherwise $L\left(s-3 / 2,\left(\pi_{i} \otimes \chi_{i}\right) \otimes \pi_{i}^{\prime \tau_{1}}, \sigma\right)$ is holomorphic at $s=5 / 2$. Also, the function $L\left(s-3 / 2,\left(\pi_{i} \otimes \chi_{i}\right) \otimes \pi_{i}^{\prime \tau_{1}}, \sigma\right)$ does not vanish for $\operatorname{Re}(s) \geqslant 5 / 2$ and satisfies a functional equation.

Since the CM-weight of $\theta$ is $\left(\frac{1}{2}, \ldots, \frac{1}{2}, \frac{-1}{2}, \ldots, \frac{-1}{2}\right)$, we see easily that it's impossible to have $\theta_{i}^{\prime \tau_{1}} \mid I_{M F_{i}}=\left(\theta_{i} \chi_{i}^{\prime \prime}\right)^{-1}$ or $\theta_{i}^{\prime \tau_{1}} \mid I_{M F_{i}}=\left(\theta_{i}^{\alpha} \chi_{i}^{\prime \prime}\right)^{-1}$ and we obtain that the function $L\left(s-3 / 2,\left(\pi_{i} \otimes \chi_{i}\right) \otimes \pi_{i}^{\prime \tau_{1}}, \sigma\right)$ is holomorphic at $s=5 / 2$.

The cases ii) and iii) are similar to the case i).

In the case iv), a), we know that $[F: E]=3, F$ is Galois over $E, \tau_{1}$ is the non-trivial automorphism of $F$ over $E$ and $\rho(\pi)^{s s}$ is a subrepresentation of

$$
\operatorname{Ind}_{\Gamma_{F}}^{\Gamma_{E}}\left(\rho_{\pi} \otimes \rho_{\pi}^{\tau_{1}} \otimes \rho_{\pi}^{\tau_{1}^{2}}\right)
$$

which verifies

$$
\left.\rho(\pi)^{s s}\right|_{\Gamma_{F}} \cong \rho_{\pi} \otimes \rho_{\pi}^{\tau_{1}} \otimes \rho_{\pi}^{\tau_{1}^{2}}
$$

We distinguish two subcases: 
1) $F \subseteq F_{i}$. Then

$$
\left.\left.\left.\left.\rho(\pi)^{s s}\right|_{\Gamma_{F_{i}}} \cong \rho_{\pi}\right|_{\Gamma_{F_{i}}} \otimes \rho_{\pi}\right|_{\Gamma_{F_{i}}} ^{\tau_{1}} \otimes \rho_{\pi}\right|_{\Gamma_{F_{i}}} ^{\tau_{1}^{2}},
$$

and we are in the same situation as in the case i) above.

2) $F \nsubseteq F_{i}$. Then

$$
L\left(s,\left.\rho(\pi)^{s s}\right|_{\Gamma_{F_{i}}} \otimes \chi_{i}\right)=L\left(s-3 / 2, \pi_{i}^{\prime} \otimes \chi_{i}^{\prime}, \sigma\right) .
$$

From Propositions 5.4 and 5.5, we get that the order of the pole of $L(s-$ $\left.3 / 2, \pi_{i}^{\prime} \otimes \chi_{i}^{\prime}, \sigma\right)$ at $s=5 / 2$ is equal to 1 if $\left.\theta_{i}^{\prime} \chi_{i}^{\prime \prime \prime}\right|_{I_{F_{i} M^{\prime}}}=1$ or $\left.\theta_{i}^{\prime \alpha} \chi_{i}^{\prime \prime \prime}\right|_{I_{F_{i} M^{\prime}}}=1$, where $\theta_{i}^{\prime}=\left.\theta_{i}\right|_{\Gamma_{M F_{i}}}, \chi_{i}^{\prime \prime \prime}=\left.\chi_{i}^{\prime}\right|_{\Gamma_{M F_{i}}}$ and $M^{\prime}$ is the quadratic extension of $E$ such that $M=M^{\prime} F$. Otherwise $L\left(s-3 / 2, \pi_{i}^{\prime} \otimes \chi_{i}^{\prime}, \sigma\right)$ is holomorphic at $s=5 / 2$. Also, the function $L\left(s-3 / 2, \pi_{i}^{\prime} \otimes \chi_{i}^{\prime}, \sigma\right)$ does not vanish for $\operatorname{Re}(s) \geqslant 5 / 2$ and satisfies a functional equation.

One could see easily that none of the characters $\left.\theta_{i}^{\prime} \chi_{i}^{\prime \prime \prime}\right|_{I_{F_{i} M^{\prime}}}$ or $\left.\theta_{i}^{\prime \alpha} \chi_{i}^{\prime \prime \prime}\right|_{I_{F_{i} M^{\prime}}}$ could have parallel weight $(0, \ldots, 0)$ and thus these characters are different from 1 and we get that $L\left(s-3 / 2, \pi_{i}^{\prime} \otimes \chi_{i}^{\prime}, \sigma\right)$ is holomorphic at $s=5 / 2$.

The case iv), b), 1) is similar to the case i), a); the case iv), b), 2) is similar to the case i, b), 2); the cases iv), b), 3) and iv), b), 4) and iv), b), 5) are similar to the case i), b).

From this section and Section 6.1, we deduce Theorem 6.1.

Remark 6.2. The results in this article remain true if we replace the prime ideal $\wp$ of $O_{F}$ by an arbitrary ideal $\mathbf{n}$ of $O_{F}$ such that each place $\wp \mid \mathbf{n}$ is unramified in $D$.

\section{REFERENCES}

[B] D. Blasius, Hilbert modular forms and the Ramanujan conjecture, preprint.

[BR] D. Blasius and J. D. Rogawski, Zeta functions of Shimura varieties, Motives, AMS Proc. Symp. Pure Math. 55, Part 2.

[C] H. Carayol, Sur la mauvaise réduction des courbes de Shimura, Compositio Mathematica, 59 (1986), nr. 2, 151-230.

[HLR] G. Harder, R. P. Langlands and M. Rapoport, Algebraische Zyclen auf HilbertBlumenthal-Flächen, J. Reine Angew. Math., 366 (1986), 53-120.

[I] T. Ikeda, On the location of poles of the triple L-functions, Compositio Math., 83 (1992), 187-237. 
[RT] J. D. Rogawski and J. B. Tunnell, On Artin L-functions associated to Hilbert modular forms of weight one, Inventiones Mathematicae, 74 (1983), 1-43.

[SE] J.-P. Serre, Linear representations of finite groups, Springer, 1977.

[T] R. Taylor, On Galois representations associated to Hilbert modular forms, Inventiones Matematicae, 98 (1989), 265-280.

[V] C. Virdol, Non-solvable base change for Hilbert modular representations and zeta functions of twisted quaternionic Shimura varieties, preprint; see my web page: http://www. math.columbia.edu/ virdol/.

Department of Mathematics

Columbia University

2960 Broadway

New York, NY 10027

U.S.A. 